\title{
Research on Cell ID Blind Detection Algorithm and ASIC Implementation in LTE - A System
}

\author{
Youqi Shen* and Guiyong Li \\ College of Communication and Information Engineering, Chongqing University of \\ Posts and Telecommunications, Chongqing 400065, China \\ syqsnail1128@126.com
}

\begin{abstract}
LTE-A system, the UE and the eNodeB cell synchronization process is very important, it can detect the physical layer cell ID and access to time and frequency synchronization, and then process data communication. The key to cell synchronization is to acquire the cell ID. The ID of the cell can be obtained by ID blind detection, and the cell system information can be obtained. Therefore, it is very important to get the cell ID quickly and accurately. With the constant updating of the protocol, the design of the user's receiving end is also constantly updated. This paper proposes a reasonable and feasible ASIC design scheme and valid UVM (Universal Verification Methodology) verification platform from the perspective of studying the cell ID blind detection algorithm as well hardware implementation by balancing the fast accuracy of data operation with hardware cost, area and power consumption, it has important practical significance. Compared with the same module of other design, ASIC design of this area is $8.8 \%$ lower, 9.3\% lower power consumption than the other design.
\end{abstract}

Key words: Cell ID blind detection; ASIC implementation; UVM verification

\section{Introduction}

With the LTE-A system of large-scale commercial, for different emerging markets, the user design of the receiver is constantly updated. In the cell synchronization process, the $\mathrm{UE}$ and the eNodeB are obtained to get time and frequency synchronization and cell ID, which are mainly divided into primary synchronization signal (PSS) detection and secondary synchronization signal (SSS) detection [1]. For the PSS detection algorithm, literature [2] gives an algorithm with low computational complexity. The algorithm first obtains the position of the PSS according to the symmetry of the PSS, and then cross-correlates with the local PSS to obtain the ID number. For the SSS detection algorithm, literature [3] compared the SSS detection algorithm in time and frequency domain, through simulation, SSS detection algorithm performance is relatively better in the frequency domain. In literature $[4,5]$, an algorithm using Hadamar matrix instead of correlation algorithm is proposed, which can reduce the complexity of SSS detection algorithm.

In this paper, through the algorithm of blind detection of cell ID, ASIC design is compared with the traditional Field Programmable Gate Array (FPGA) processing, the ASIC design is more suitable for real-time and reliability requirements of large-scale and complex digital signal processing, cost, power consumption, etc. At the same time, through the verification of hardware and algorithm performance, the design module has better performance in area and power consumption than the same kind of design module through the verification of UVM. So it can meet and apply to LTE-A user terminal and has certain practical significance. 


\section{Related Algorithms Research}

\subsection{PSS Coarse Synchronization}

PSS coarse synchronization is to quickly find the approximate location of PSS and get the cell group ID number $N_{I D}^{(2)}$. Since the PSS has a period of $5 \mathrm{~ms}$ and each field contains PSS and the position is fixed, it is possible to take the data in two consecutive fields and slide the correlation data between the two sets of received data. The position of the maximum value is the approximate position of PSS, The received PSS is associated with the local PSS according to the approximate location of the PSS to obtain the $N_{I D}^{(2)}$.

The specific steps of the program are: receive a frame of data, divided into two fields, respectively, using $r_{1}(n)$ and $r_{2}(n)$ to represent (assuming that each field data contains a complete PSS), the two sets of receive data. And the correlation function is given by:

$C(d)=\sum_{n=0}^{N-1} r_{1}(d+n) r_{2}^{*}(d+n)$

Where $N$ denotes the length of the correlation window, that is, an OFDM symbol length. When the down sampling rate is set to $1 / 16, N$ takes $128 ; d$ indicates the sliding distance, and the sliding range is $[0,9471]$. The average energy of the two groups of received data is given by:

$$
R(d)=\frac{1}{2} \sum_{n=0}^{N-1}\left(\left|r_{1}(d+n)\right|^{2}+\left|r_{2}(d+n)\right|^{2}\right)
$$

The timing measure function is:

$T M(d)=\frac{|C(d)|^{2}}{|R(d)|^{2}}$

The approximate position of PSS can be obtained as:

$$
\hat{d}=\arg \max _{d}\{T M(d)\}
$$

\subsection{PSS Fine Synchronization}

PSS coarse synchronization is only to obtain the approximate position of PSS, the accuracy position cannot meet the requirements, need to carry out PSS fine synchronous operation. In this paper, the algorithm based on receiving PSS and local PSS is adopted.

First, an $N_{I D}^{(2)}$ obtained by coarse synchronization of the PSS is locally generated in the frequency domain PSS, and then transformed into the time domain by the IFFT. Then a series of values are obtained by sliding correlation calculation in the range of 64 points around $\hat{d}$, i.e., $\left[\hat{d}^{*} 16-64, \hat{d}^{*} 16+63\right]$, and then the time-domain received data before descending. The position where the maximum value is located is PSS fine synchronous position. The correlation function is as follows:

$$
C(d)=\sum_{n=d^{*} 16-64}^{\hat{d} * 16+63}\left|r(d+n) s^{*}(n)\right|^{2}
$$


The position of the maximum value obtained by Eq. (5) is the position of the fine synchronization:

$$
d_{P S S}=\underset{d}{\arg \max }\{C(d)\}
$$

\subsection{SSS Detections}

After the PSS synchronization is completed, the exact position of the PSS is determined. However, it cannot be determined whether the current data is the first or second field. The SSS takes $10 \mathrm{~ms}$ as the cycle, so it can detect the cell group ID $N_{I D}^{(1)}$. In order to reduce the complexity and reduce the computational complexity, the parameters $m_{0}$ and $m_{1}$ are obtained by descrambling. According to the one-to-one correspondence between $m_{0}$ and $m_{1}$ and $N_{I D}^{(1)}$, the cell ID group number $N_{I D}^{(1)}$. In this paper, we use the coherent detection algorithm [6].

Since the SSS detection is performed after the PSS detection, when the channel coherence time is greater than 4 OFDM symbol lengths, the PSS is used to obtain the estimated value $\hat{H}_{P S S}(k)$ of the channel impulse response, and the frame synchronization is performed by the coherent detection method.

The received time domain PSS is transformed into the frequency domain PSS by the FFT transformation, and is denoted by $R_{P S S}(k)$, and the local frequency domain PSS is generated, denoted by $T_{P S S}(k)$. If the channel coherence time is greater than 4 OFDM symbols, the channel impulse response estimate can be obtained:

$$
\hat{H}_{P S S}(k)=R_{P S S}(k) / T_{P S S}(k)
$$

According to the PSS position, the time-domain SSS is transformed into the frequency-domain SSS by FFT, and then the channel compensation is performed:

$$
\hat{R}_{S S S}(k)=R_{S S S}(k) \hat{H}_{P S S}(k)
$$

And divides $\hat{R}_{S S S}(k)$ into a sequence $\hat{R}_{S S S}(2 k), \hat{R}_{S S S}(2 k+1)$ consisting of even and odd-numbered bits. According to the PSS synchronization obtained by the $N_{I D}^{(2)}$ and scrambling sequence $c_{0}(k)$, the $\hat{R}_{S S S}(2 k)$ to descramble:

$a_{m 0}(k)=\hat{R}_{S S S}(2 k) c_{0}(k)$

The different cyclic shift sequences $S^{(i)}(k)$ of $a_{m 0}(k)$ and $m$ sequences are segmented and the estimated value $m_{0}$ is obtained according to the maximum value $\hat{m}_{0}$ :

$$
\hat{m}_{0}=\underset{i}{\arg \max }\left\{\sum_{j=0}^{M-1}\left|\sum_{k=j N_{M}}^{(j+1) N_{M}} a_{m_{0}}(k) S^{(i)}(k)\right|^{2}\right\}
$$

The above formula $i=0,1, \ldots, 30, M$ is the number of segments, $N_{M}$ is the data length, the above formula $M=4$.

Generate a local scrambling code $c_{1}(k)$ and $z_{1}^{\left(\hat{m}_{0}\right)}(k)$ two sequences, the $\hat{R}_{S S S}(2 k+1)$ descrambling operation, too: 
$a_{m 1}(k)=\hat{R}_{S S S}(2 k+1) c_{1}(k) z_{1}^{\left(\hat{m}_{0}\right)}(k)$

$m_{1}$ estimate of $\hat{m}_{1}$ and $\hat{m}_{0}$ similar, not repeat them:

$\hat{m}_{1}=\underset{i}{\arg \max }\left\{\sum_{j=0}^{M-1}\left|\sum_{k=j N_{M}}^{(j+1) N_{M}} a_{m 1}(k) S^{(i)}(k)\right|^{2}\right\}$

The correspondence between $m_{1}$ and $m_{0}$ estimates $\hat{m}_{0}$ is as follows:

$\begin{cases}m_{1} \in\left[0,1, \cdots, \hat{m}_{0}+7\right], & \text { when } \hat{m}_{0} \in[0, \cdots, 2], \text { and } m_{1} \neq \hat{m}_{0} \\ m_{1} \in\left[0,1, \cdots, \hat{m}_{0}+6\right], & \text { when } \hat{m}_{0} \in[3, \cdots, 7], \text { and } m_{1} \neq \hat{m}_{0} \\ m_{1} \in\left[\hat{m}_{0}-7, \cdots, \hat{m}_{0}+6\right], & \text { when } \hat{m}_{0} \in[8,9], \text { and } m_{1} \neq \hat{m}_{0} \\ m_{1} \in\left[\hat{m}_{0}-6, \cdots, \hat{m}_{0}+6\right], & \text { when } \hat{m}_{0} \in[10, \cdots 24], \text { and } m_{1} \neq \hat{m}_{0} \\ m_{1} \in\left[\hat{m}_{0}-6, \cdots, 30\right], & \text { when } \hat{m}_{0} \in[25, \cdots 30], \text { and } m_{1} \neq \hat{m}_{0}\end{cases}$

Can be obtained, $\hat{m}_{0}$ is less than $\hat{m}_{1}$, the receiving SSS is in subframe 0 . Otherwise in the subframe 5 .

Let $M=\hat{m}_{0}-\hat{m}_{1}$, then $N_{I D}^{(1)}$ can be obtained by the following formula:

$N_{I D}^{(1)}=30(M-1)+\hat{m}_{0}-(M-1)(M-2) / 2$

Using the descrambling coherent detection algorithm, a total of 44 correlation operations (estimated $m_{0}, 30$ times; estimate $m_{1}, 14$ times).

\section{ASIC Design and Implementation}

\subsection{Functional Structure}

The cell synchronization ID blind detection module includes a control module (ctrl), an interface module (regif), a memory module (mem) and an arithmetic processing module (cal). The external interaction with AXI Direct memory access (AXIDMA) triggers the reading and writing of Double Data Rate (DDR) data.

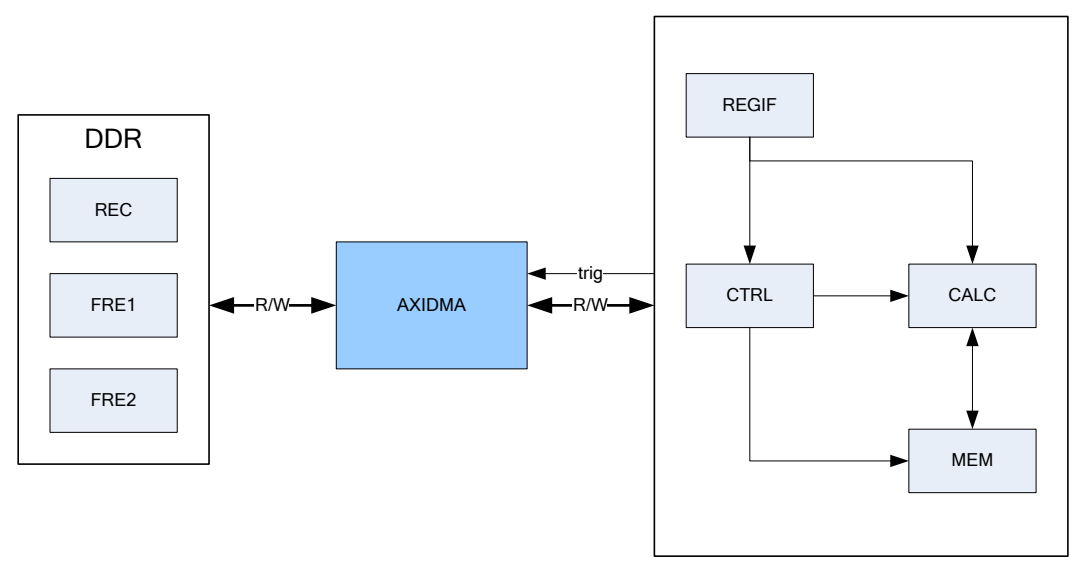

Figure 1. Block Diagram of the Functional Block Structure

\subsubsection{Control Module}

In this paper, the control module with finite state machine control, the jump as follows: 


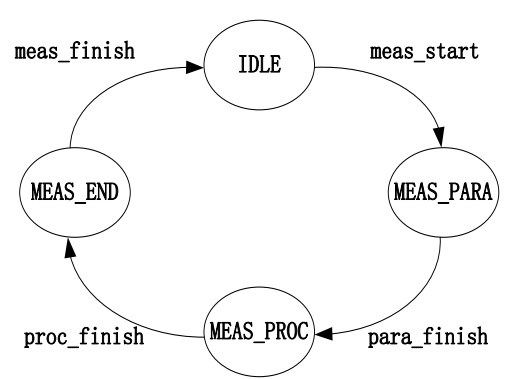

Figure 2. Module State Machine Control Transfer Diagram

1. IDLE state: not activated are in this state, when meas_start is valid, IDLE state jumps to MEAS_PARA state.

2. MEAS_PARA state: After entering this state, when the register parameter is read, para_finish is set to 1 , indicating that the parameter reading is complete and the MEAS_PARA state jumps to the MEAS_PROC state.

3. MEAS_PROC state: enter the state, the specific functions of the module operation. When the operation is complete, the proc_finish signal is set to indicate that the operation has been completed and the MEAS_PROC state jumps to the MEAS_END state.

4. MEAS_END state: When the module enters this state, it indicates that the external configuration of the functional tasks have been completed, meas_finish signal is set to jump from the MEAS_END state to IDLE state.

\subsubsection{Interface Module}

The interface module uses the common ZSP bus interface, sets up the parameter which needs to the module correlation function.

Table 1. Interface Signal List

\begin{tabular}{|c|c|c|}
\hline Signal name & Direction & Description \\
\hline \multicolumn{3}{|l|}{ Clock and reset signal } \\
\hline clk & Input & Function clock \\
\hline rst_ & Input & Function reset, active low \\
\hline $\mathrm{zclk}$ & Input & Interface clock \\
\hline zrst__ & Input & Interface reset, active low \\
\hline \multicolumn{3}{|l|}{ ZSP interface signals } \\
\hline $\mathrm{zcs}_{-}$ & Input & ZSP bus chip select, active-low \\
\hline ziosel & Input & $\begin{array}{l}\text { ZSP bus register selection, high-level access to the register, } \\
\text { low-level access to memory }\end{array}$ \\
\hline zwr_[1:0] & Input & $\begin{array}{l}\text { ZSP bus write enable, active low; zwr_[0] is low: Low } \\
\text { 32-bit write enable; zwr_[1] is low: High 32-bit write } \\
\text { enable; zwr_[1:0] is low: 64-bit write enable }\end{array}$ \\
\hline zrd_ & Input & ZSP bus read enable, active low \\
\hline $\operatorname{zaddr}[11: 0]$ & Input & ZSP bus address \\
\hline zrdata[63:0] & Output & ZSP bus read data \\
\hline zwdata[63:0] & Input & ZSP bus write data \\
\hline \multicolumn{3}{|l|}{ Other signals } \\
\hline dma_start & Input & $\begin{array}{l}\text { Synchronous with the interface clock sclk, the rising edge of } \\
\text { the hardware module. }\end{array}$ \\
\hline fft_irq & Output & $\begin{array}{l}\text { Interrupt signal, active-high. Synchronized with the } \\
\text { interface clock sclk, high level to maintain } 32 \mathrm{zclk} \text { clock } \\
\text { cycle. }\end{array}$ \\
\hline clk_en & Input & $\begin{array}{l}\text { Memory clock enable signal. When the module is idle and } \\
\text { the external memory is not accessed, pulling this signal low } \\
\text { can reduce the module memory power consumption. }\end{array}$ \\
\hline
\end{tabular}


Interface module in addition to the need to design interface signals, but also need to design parameter registers, easy ZSP bus to read and write, to achieve different functional module configuration.

Table 2. Module Register List

\begin{tabular}{|c|c|}
\hline Name & Description \\
\hline MEAS_FFF_CTRL & Control register \\
\hline FFT_PARA & FFT parameter register \\
\hline FRE_PARA & $\begin{array}{l}\text { Frequency offset compensation parameter } \\
\text { register }\end{array}$ \\
\hline GEN_PARA & $\begin{array}{l}\text { Produces a local PSS or SSS parameter } \\
\text { register }\end{array}$ \\
\hline MAX_PARA & $\begin{array}{l}\text { Maximum value lookup parameter } \\
\text { register }\end{array}$ \\
\hline PSS_H_PARA & $\begin{array}{l}\text { PSS impulse response calculation } \\
\text { parameter register }\end{array}$ \\
\hline M0_PARA & M0 Value estimation parameter register \\
\hline M1_PARA & M1 Value estimation parameter register \\
\hline MEAS_FFF_INTF & Interrupt Flag Register \\
\hline FFT_MAX_GENE & $\begin{array}{l}\text { Maximum and normalization factor } \\
\text { registers }\end{array}$ \\
\hline
\end{tabular}

\subsubsection{Memory Module}

In this paper, the design of the module, a total of 8 memory, each memory size are Spram128x64bw, both as input and output memory. According to the different functions of the module, the specific allocation of the use of eight blocks memory.

\subsubsection{Arithmetic Processing Module}

This module mainly implements PSS coarse synchronization, PSS fine synchronization, SSS blind detection in the computing processing unit. As follows:

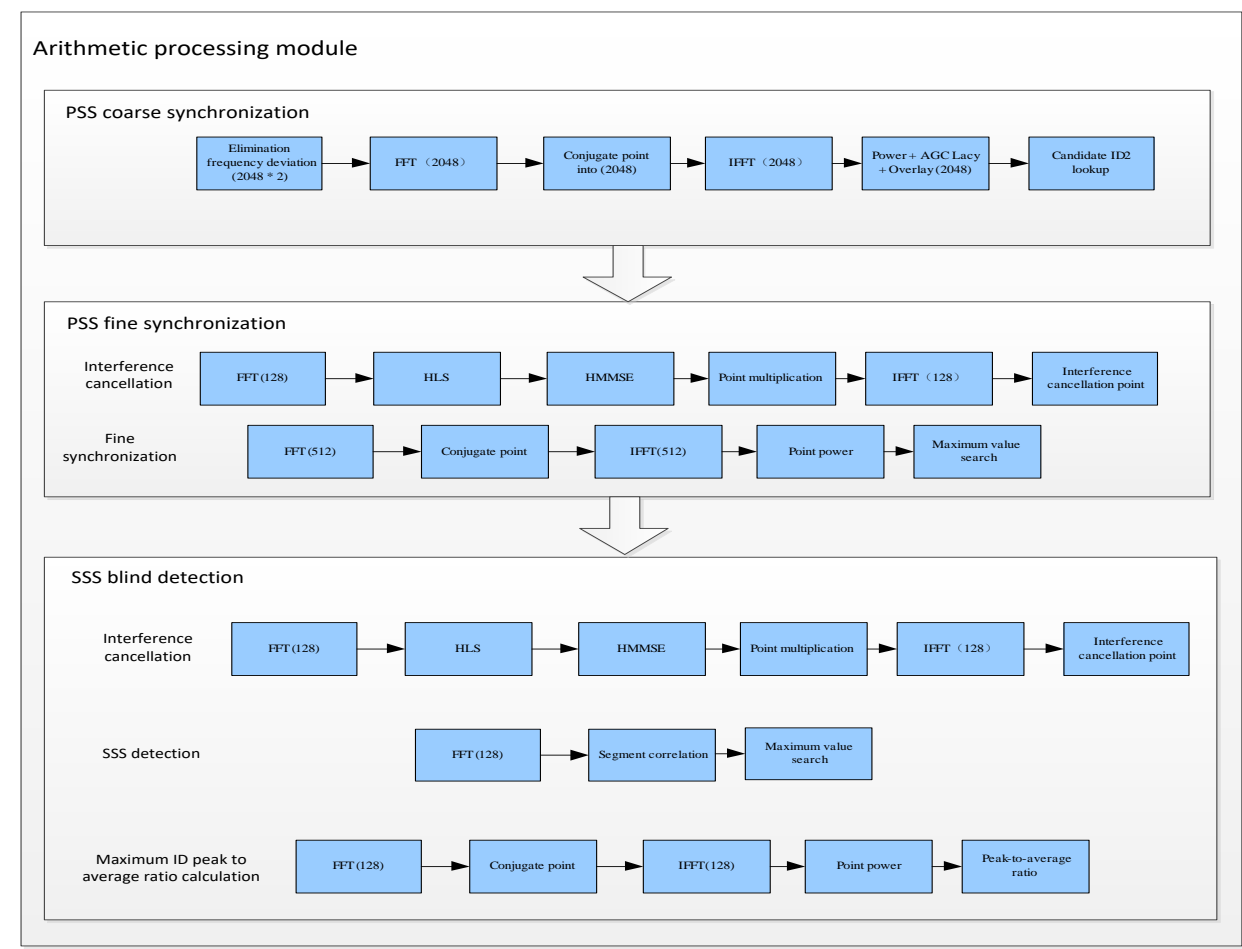

Figure 3. Module Operation Processing Unit Flow Chart 


\subsection{Hardware Implementation Process}

ID blind detection hardware implementation steps and the corresponding storage direction as shown below:

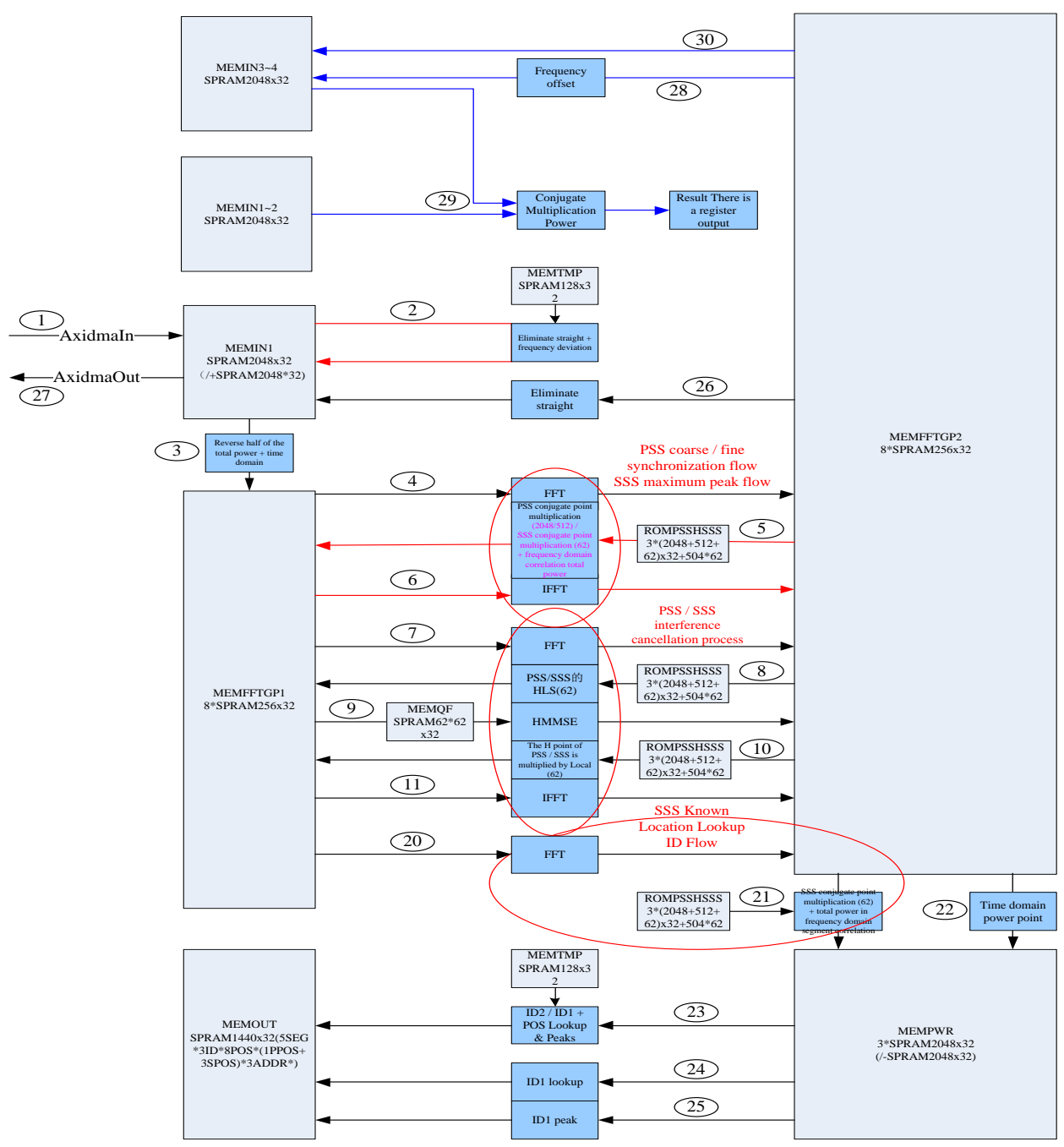

Figure 4. Module Hardware Implementation Flow Diagram

PSS coarse synchronization: steps $1,2,27,3,4,5,6,22,23$;

PSS fine synchronization: steps $1,3,4,5,6,22,23$;

PSS interference cancellation: steps 1, 3, 7, 8, 9, 10, 11, 26, 27;

PSS known $N_{I D}^{(2)}$ coarse synchronization: steps 1, 2, 27, 3, 4, 5, 6, 22, 23;

SSS known position finding $N_{I D}^{(1)}$ : steps 1, 3, 20, 21, 24;

SSS maximum $N_{I D}^{(1)}$ peak average calculation: steps 1, 3, 4, 5, 6, 22, 25;

SSS interference cancellation: steps $1,3,7,8,9,10,11,26,27$;

SSS known position and ID pair ID order: steps 1, 3, 20, 21, 24;

\subsection{RTL Code to Achieve Results}

According to the above algorithm research and hardware implementation flow, Resistor Transistor Logic (RTL) code is written, the ASIC implementation of the module 
is completed. After the code is compiled, it is checked by VCS simulation tool for its correctness and feasibility ${ }^{[7]}$. The code inspection report is shown below.

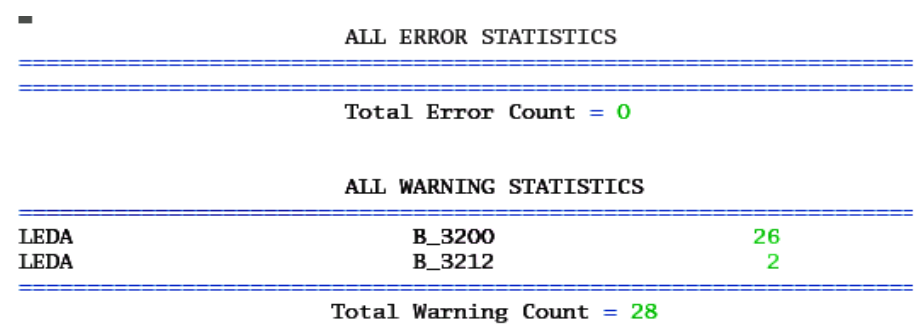

Figure 5. RTL Code Inspection Report

In this paper, the use of Verdi simulation tool RTL code for the module was integrated, this will help to check the code whether it can be integrated and normative, integrated top-level structure shown below. The top-layer structure mainly comprises a control module meas_fft_ctrl, an interface module meas_fft_regif, a memory module meas_fft_mem, a butterfly operation unit meas_fft_proc, a frame synchronization and a cell group ID number detection function module meas_fft_sss, and a frequency offset compensation function module meas_fft_fre.

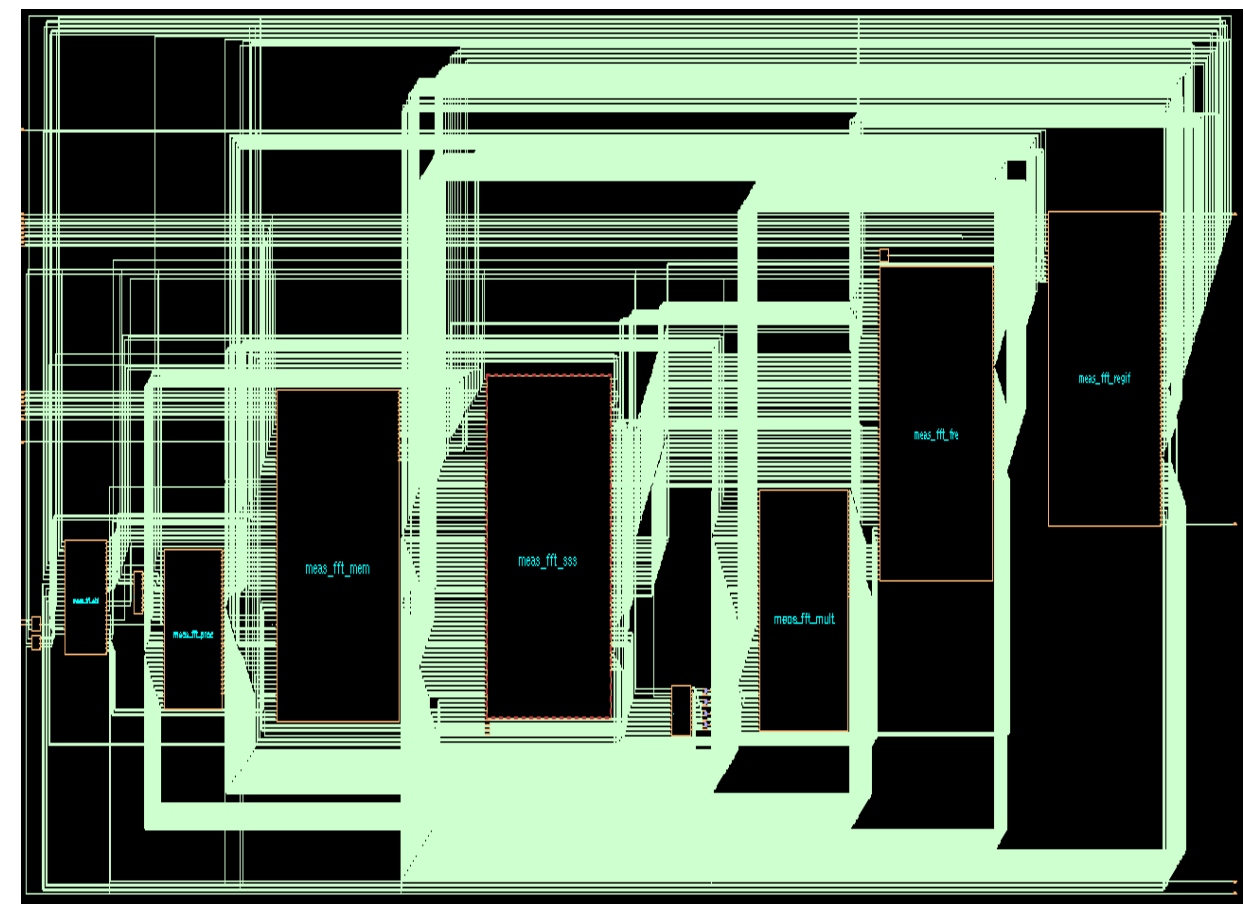

Figure 6. Top Layer Circuit Diagram

RTL code designed to achieve the function of the target, but also the need for functional verification.

\section{UVM Simulation Verification}

The verification platform of ID-based blind inspection module is mainly based on the UVM authentication method of SystemVerilog verification language, which mainly consists of two parts: random verification environment and reference model [8].

The block diagram of the entire verification platform for the module is shown below. 


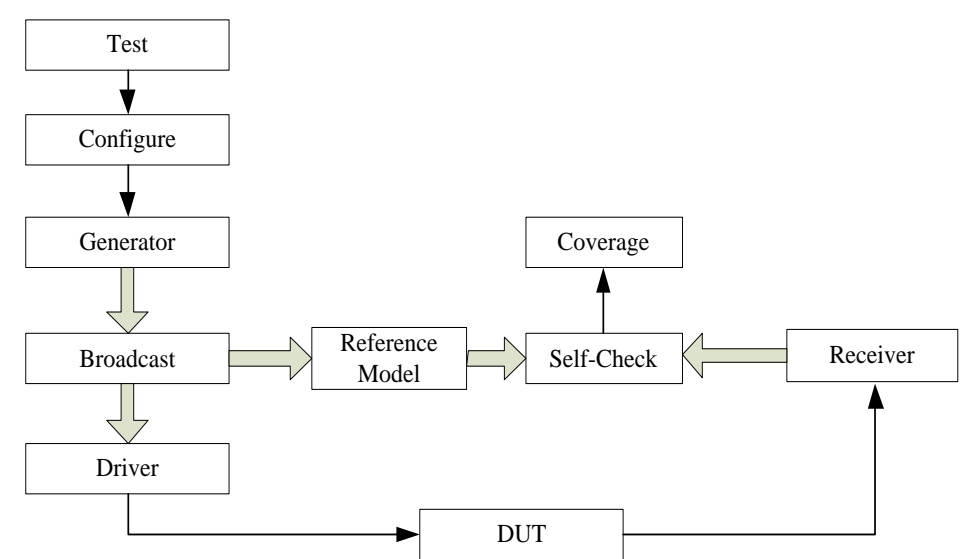

Figure 7. Module Verification Platform Structure

\subsection{Simulation and Verification Results}

This section use VCS simulation tools and graphical debugging tools, the module was a comprehensive functional verification, the verification process need to cover all the function points, simulation waveform more, so we select several typical waveform analysis instructions.

\subsubsection{Register Basic Attribute Simulation Results}

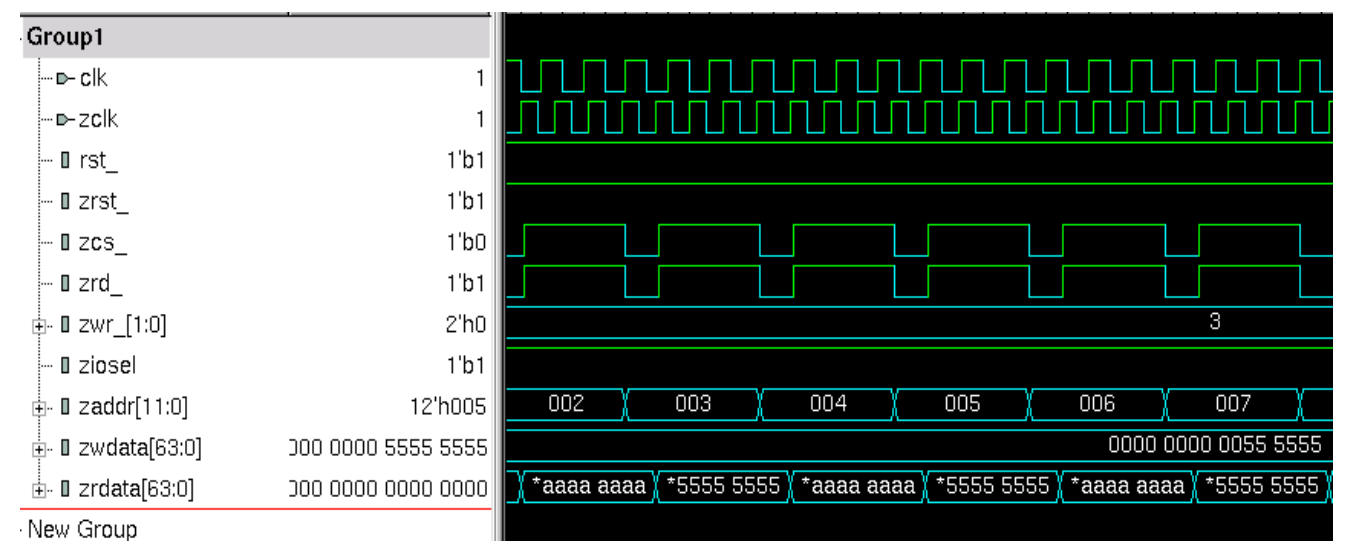

Figure 8. Interface Timing and Write Register Waveform

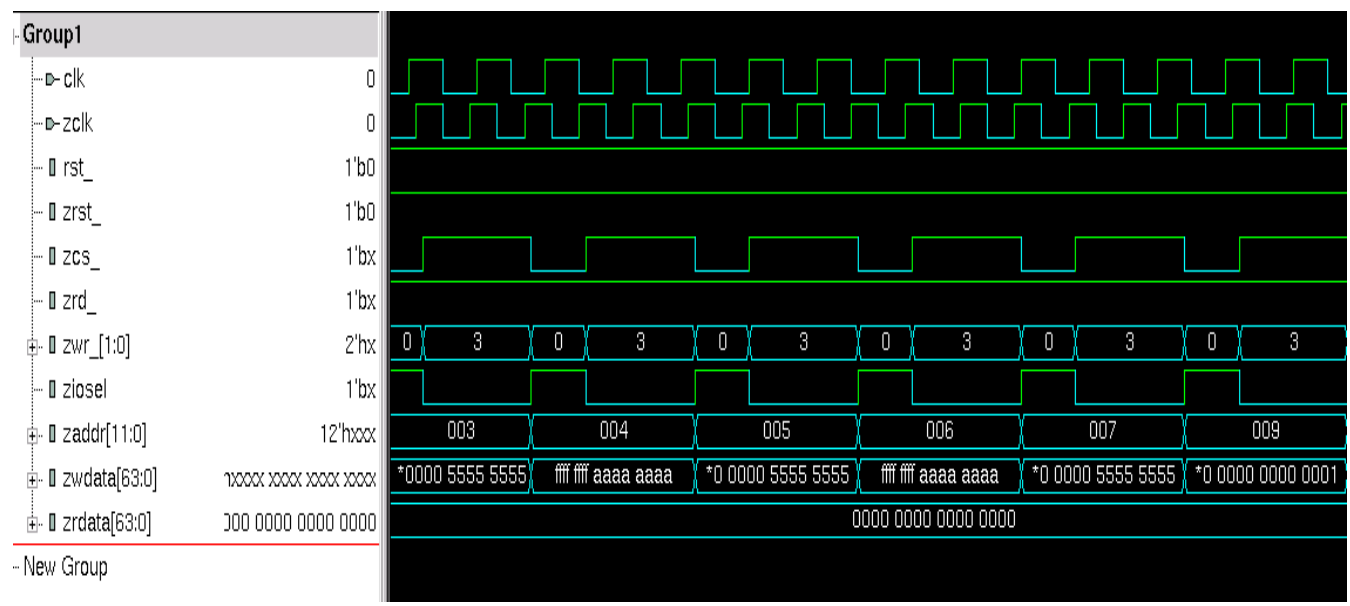

Figure 9. Register Reads the Waveform 
It can be seen from the figure, the module to meet the bus interface timing; the module's registers can be normal reset, and the reset value is correct; the module's registers can read and write, and read and write attributes fully meet the design intent.

\subsubsection{M0 Value Estimation Function Simulation Results}

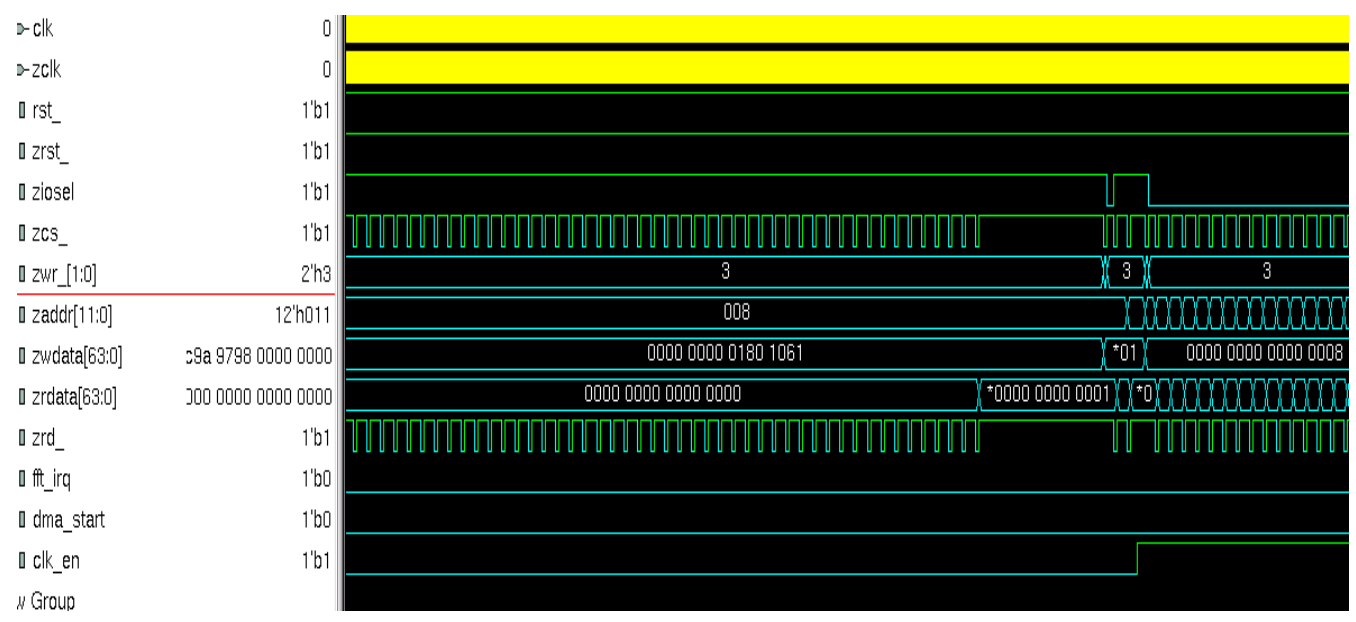

Figure 10. M0 Value Estimation Function Simulation Waveform

As can be seen from the figure, this simulation does not enable interrupts, so after the end of the module operation, and no interrupt signal. In this case, the value of the interrupt flag register can only be read until the interrupt flag register is set to determine that the module has finished running, and then read the output data from the memory and compare the correctness of the output data. The results show that this function can achieve the desired goal.

\subsubsection{M1 Value Estimation Function Simulation Results}

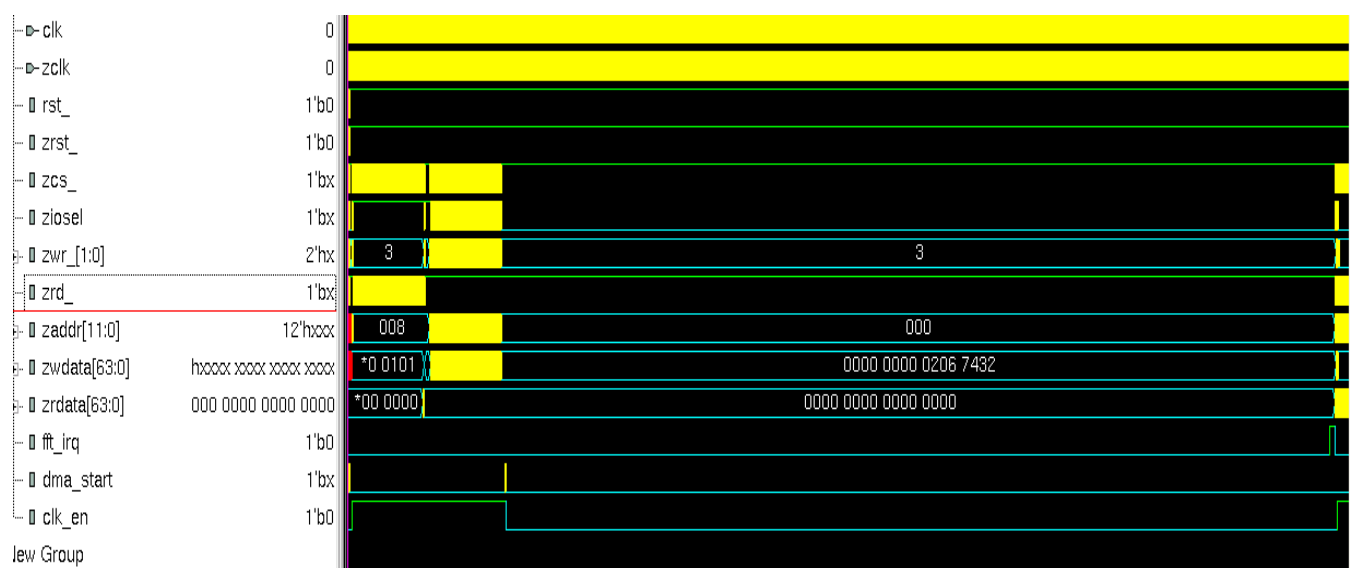

Figure 11. M1 Value Estimation Function Simulation Waveform

Figure 11 shows the simulation waveform for the M1 value estimation function. From the waveform can be seen, this simulation is by the DMA boot module work. After the module runs, the interrupt signal is normally generated. Comparison of the data after the simulation shows that the function can achieve the desired goal. 


\subsection{Simulation Results Analysis}

By observing the simulation waveforms and emulation log information of each test case, it can be concluded that the registers and the memory can be read and written normally, and there is no data bit sticking or address error, etc. The bus operation is normal and there is no bus which cannot be read and written. The actual working time of the module is shown in Table 3, and the actual working time is in accordance with the time evaluation in the program; the interrupt can be generated normally; each function of the module can achieve the expected target.

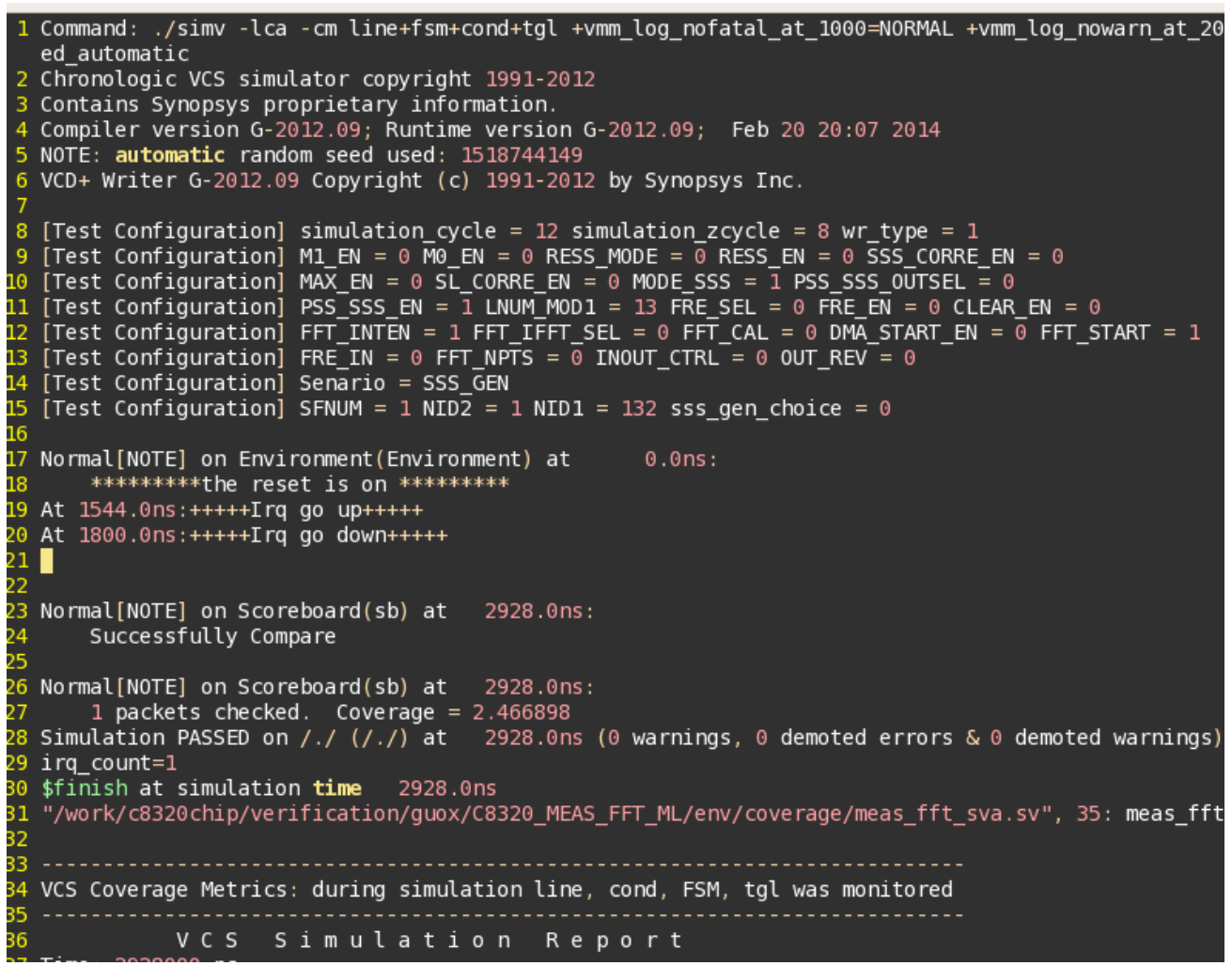

Figure 12. Module Emulation Log Information

Table 3. Lists the Module Evaluation Time Versus the Actual Working Time

\begin{tabular}{lll}
\hline & Evaluation time & real time \\
\hline FFT (2048 points) & 3000 cycles & 2934 cycles \\
Generates a local PSS & 384 cycles & 384 cycles \\
A local SSS is generated & 384 cycles & 384 cycles \\
Maximum value search & 9544 cycles & 9329 cycles \\
M0 value estimation & 768 cycles & 683 cycles \\
M1 value estimation & 896 cycles & 873 cycles \\
\hline
\end{tabular}

\subsection{Logic Synthesis}

In this paper, the use of DC synthesis tools on the logic of the module synthesis, it can RTL code into the gate-level netlist, and produce the appropriate logical file reports.

In order to effectively reduce power consumption and reduce the chip area, this paper uses a pre-insert with the scanning side of the latch type integrated gate clock. The results are shown below. 


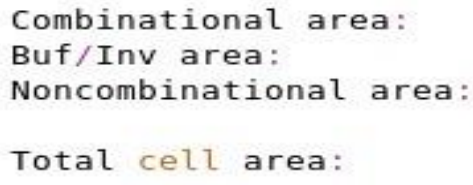

Figure 13. Module Area Report

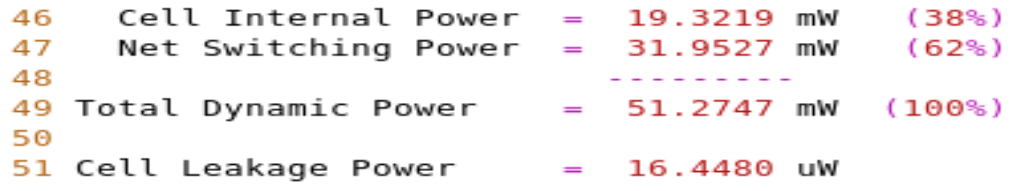

Figure 14. Module Power Report

The following conclusions can be drawn from Figure 13 and Figure 14:

1 , the design of the integrated logic area is $1302392.271666 \mathrm{um} 2$, where the combinational logic area is $299699.322752 \mathrm{um} 2$, the sequential logic area is 1002 722.948914 um2;

2 , the design of the operating voltage is 0.99 , dynamic power consumption is 51.274 $7 \mathrm{mw}$, the static power consumption is $16.448 \mathrm{uw}$, the total power consumption is 51.291 $1 \mathrm{mw}$.

\section{Conclusion}

The logical area of the synchronous ID blind detection module in LTE-A system designed by this paper is $125669.429722 \mathrm{um} 2$, about $8.8 \%$ smaller than that of the same module. The power consumption of the module is $5.2592 \mathrm{mw}$, about $9.3 \%$ lower than that of the same module. It can be seen, in line with this without sacrificing the premise of the larger performance of the hardware area, power consumption considerations, compared with similar modules designed to have certain advantages, to meet the design requirements, with application value.

\section{References}

[1] A. Andreescu, A. Ghita and A. A. Enescu, "Long term evolution primary synchronization algorithms", Electronics and Telecommunications (ISETC), 2010 9th International Symposium on. IEEE, (2010), pp. $125-128$.

[2] Z. Zhang, J. Liu and K. Long, "Low-complexity cell search with fast PSS identification in LTE", Vehicular Technology, IEEE Transactions, vol. 61, no. 4, (2012), pp. 1719-1729.

[3] J. Zhang and X. Huang, "Autocorrelation based coarse timing with differential normalization", Wireless Communications, IEEE Transactions, vol. 11, no. 2, (2012), pp. 526-530.

[4] J. I. Kim, J. S. Han and H. J. Roh, "SSS detection method for initial cell search in 3GPP LTE FDD/TDD dual mode receiver", Communications and Information Technology, 2009. ISCIT 2009. 9th International Symposium on. IEEE, (2009), pp. 199-203.

[5] A. Andreescu, A. Ghita and A. A. Enescu, "Long term evolution primary synchronization algorithms", Electronics and Telecommunications (ISETC), 2010 9th International Symposium on IEEE, (2010), pp. 125-128.

[6] Y. Sheng and X. Luo, "Algorithm Study on Cell Search in LTE", Communications Technology, vol. 3, (2009), pp. 035.

[7] Synopsys. VCS User Guide Version 6.1. 2014: 12-18.

[8] M. Keaveney, A. McMahon and N. O'Keeffe, "The development of advanced verification environments using system Verilog”, Signals and Systems Conference, 208. (ISSC 2008). IET Irish. IET, (2008), pp. 325-330. 


\section{Author}

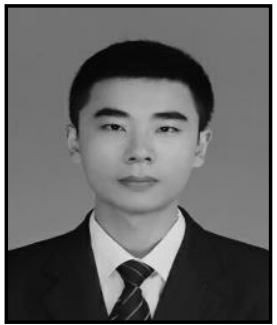

Youqi Shen, got his B.S. degree from Weifang University, and his Master degree from Chongqing University of Posts and Telecommunications, year in 2014 and now. His main research is mobile communication terminal technology. 
International Journal of Advanced Science and Technology Vol.97 (2016) 